\title{
Rire français et rire italien dans Les Plaisirs de la Tronche (1711)
}

Jean Sgard

\section{(2) OpenEdition}

\section{Journals}

\section{Édition électronique}

URL : http://journals.openedition.org/recherchestravaux/284

DOI : 10.4000/recherchestravaux.284

ISSN : 1969-6434

\section{Éditeur}

UGA Éditions/Université Grenoble Alpes

\section{Édition imprimée}

Date de publication : 30 mai 2005

Pagination : 201-209

ISBN : 0151-1874

ISSN : 0151-1874

\section{Référence électronique}

Jean Sgard, "Rire français et rire italien dans Les Plaisirs de la Tronche (1711) », Recherches \& Travaux [En ligne], 67 | 2005, mis en ligne le 30 septembre 2008, consulté le 08 septembre 2020. URL : http:// journals.openedition.org/recherchestravaux/284; DOI : https://doi.org/10.4000/recherchestravaux. 284 
Jean SGARD

Université Stendhal - Grenoble 3

\section{Rire français et rire italien dans Les Plaisirs de la Tronche (I7II)}

En février I7II était représentée à Grenoble par la Troupe italienne de Monsieur Dominique une comédie nouvelle composée par M.*** et intitulée Les Plaisirs de La Tronche. Je dois à Pierre Monnier, qui enseignait, il n'y pas si longtemps, les pratiques de mise en scène dans notre université, d'avoir découvert cette comédie dont un exemplaire imprimé subsiste à la Bibliothèque de la Ville ${ }^{\mathrm{I}}$. Cette comédie n'est pas un chef-d'œuvre, mais elle est originale, et elle nous éclaire sur un moment obscur de l'histoire du théâtre italien en France: celui qui sépare la disgrâce de la troupe italienne de Paris en I697 du retour des Italiens sous la Régence en I7I6. Michel Gilot et Jean Serroy, dans La Comédie à l'àge classique, ont souligné la richesse de l'apport italien au théâtre français au XVII ${ }^{e}$ siècle et la remarquable fécondité de la nouvelle comédie italienne de $17 \mathrm{I} 6$ à $174 \mathrm{O}^{2}$. Entre les deux troupes, la différence est profonde; seuls quelques acteurs sont passés de l'une à l'autre et le goût s'est totalement modifié en vingt ans, mais une sorte d'éclipse nous cache la nature de cette modification. On serait porté à croire qu'en I697, les Italiens sont retournés en Italie; or ce n'est pas le cas : ils sont pour la plupart restés en France; une partie d'entre eux a joué au théâtre de la Foire à Paris, une autre en province comme cette Troupe italienne de Monsieur Dominique qui jouait à Grenoble et à Lyon en I7II. Avant de parler de cette comédie, parlons un peu de M.*** et de Monsieur Dominique.

L'épître liminaire de l'édition grenobloise est signée «L. Ch... D.L.B.», que l'on a toujours interprété comme désignant un chevalier de La Barre dont

I. La pièce a été imprimée chez André Faure, place du Palais; voir l'exemplaire de la Bibliothèque municipale de Grenoble sous la cote $\mathrm{O}$ I4713.

2. Paris, Belin Sup, I997, p. 58-62, I73-I8I, 244-257. 
en fait on ignore tout ${ }^{3}$. J'observe toutefois que, trois ans auparavant, un chevalier de La Baume publiait à Grenoble, chez François Champi, La Fée bienfaisante, "Comédie composée par $\mathrm{Mr}^{* * *}$, ornée de danses $\&$ de musique", représentée dans la même ville par l'Académie royale de musique le 7 juillet 1708. Cette pièce, par le mélange de comédie et de scènes musicales qui la caractérise, évoque dans une certaine mesure Les Plaisirs de La Tronche: les deux comédies pourraient bien être sorties de la même fabrique. Cela ne nous avance d'ailleurs pas beaucoup; La Baume, qui fut organisateur des spectacles de la ville de Grenoble entre 1720 et I730, reste mal connu, et il se peut qu'il n'ait écrit aucune de ces deux comédies; mais ce n'est pas le problème qui nous retiendra ici ${ }^{4}$. Sa préface aux Plaisirs de La Tronche nous le montre très mêlé au milieu des "gens de distinction" de la ville, attentif à plaire à sa protectrice, la présidente de Saint-Ange, mais amené à écrire sur commande:

[...] La Tronche étant un des plus agréables endroits qui soit aux environs de cette Ville, \& cet aimable séjour faisant le plaisir de tout ce qu'il y a de Gens de distinction, j'ay crû, MADAME, que vous ne désaprouveriez point la liberté que je prends de vous présenter ce petit divertissement; mais comme je suis contraint d'en faire la partie avec une Troupe italienne dont le caractère m'est assez inconnu, outre que la beauté de leur jeu est dans le geste $\&$ la manière dont ils débitent ce qu'ils disent, je me suis flatté, MADAME, que tout cela excuserait les fautes que vous pourrez trouver dans le stille de leurs conversations [...]

L. B. offre donc à la veuve d'un président du parlement de Grenoble cette "petite partie de campagne» qu'il a écrite de la main gauche en collaboration avec les comédiens, un peu malgré luis. Son épître liminaire le montre surtout pressé de faire sa cour et de se donner le beau rôle. Tout porte à croire que le véritable homme de théâtre, dans cette affaire, est Dominique. Que cette représentation ait lieu en février me parait confirmer que les Italiens sont de passage pour la saison d'hiver, qui s'achève au carême, et qu'il faut profiter de leur présence. Quelle est donc cette Troupe italienne de M. Dominique? Le nom de Dominique, en I7II, ne peut désigner que Pierre François Biancolelli,

3. Conlon (Prélude au siècle des Lumières) donne La Barre, sans preuve. Le Chevalier de La Baume signe la préface de La Fée bienfaisante «Comédie. Composée par M. M***, ornée de danses et de musique. Le théâtre représente une campagne au bout de laquelle on voit le château où la fée fait son séjour. Représentée à Grenoble par l'Académie royale de musique le 7 juillet I708 ", Grenoble, François Champ, 1708 (exemplaire à la Bibliothèque de Lyon).

4. Nous préparons une édition critique des Plaisirs de La Tronche qui fera une large place à la présidente de Saint-Ange et au chevalier de La Baume-Pluvinel.

5. "Faire sa partie avec» fait allusion à une collaboration continue, comme pour une partie de cartes (Littré, «Partie», par. I4). 
fils du célèbre Dominique, le plus illustre des Arlequins, mort en I688. Dominique fils était né à Paris en $1680^{6}$. Je cite Gilot et Serroy:

Il débuta à Toulouse dans la troupe de Pascariel et mena longtemps en province, avec les Comédiens italiens, une triple carrière d'acteur, de chef de troupe et d'auteur comique. Il tint pendant plusieurs années le rôle d'Arlequin au théâtre de la Foire, avant d'être engagé, à la demande du Régent, dans la nouvelle troupe de Luigi Riccoboni. Il y débuta en Pierrot en octobre I7I7 [...]7

Léris, dans son Dictionnaire portatif du théâtre, précise qu'il épousa la fille de Pascariel, se rendit en Italie, puis revint en France et « joua pendant quelque temps dans les provinces». Quelques dates feraient mieux notre affaire ${ }^{8}$. À défaut, on peut chercher sa trace dans le Calendrier des spectacles de l'ancien régime établi par Barry Russel ${ }^{9}$ : une troupe italienne fait son apparition à Lyon en 1703 et joue dans la salle de l'Opéra de Belle-Cour la Vengeance de Colombine ou Arlequin beau-frère du grand Turc de Nicolas Barbier : ce dernier parait bien avoir écrit pour le couple Arlequin-Colombine, donc pour la famille Biancolelli ${ }^{\circ}$. En 1704, Dominique donne à la cour de Lunéville Les Amours d'Arlequin et La Fête galante. Après deux ans de silence, on voit des "Comédiens italiens privilégiés de Mgr le Maréchal de Villeroy» donner à Lyon L'Opéra interrompu. En I709, Dominique est à Rennes où il donne, avec la Troupe des comédiens italiens La Promenade de Rennes. En I7IO, le voici de retour à Lyon où il joue coup sur coup La Femme infidèle ou Les Apparences trompeuses, L'Heureux Naufrage et Les Soirées d'été; en I7II, son port d'attache étant toujours Lyon et plus précisément la salle de Belle-Cour, il donne Les Amants esclaves; en I7I2, il représentera encore La Promenade des Terreaux, et après un voyage à Toulouse où il donne La Fausse Belle-mère, il revient à Lyon, puis à Grenoble, où est imprimé et sans doute représenté en 1713 Le Procès des comédiens français et italiens. On note encore en I7I3 un séjour à Bordeaux, pour Les Salinières; après quoi, sa carrière devient parisienne. Il s'était rendu

6. Taviani, dans Il segreto della commedia dell'arte, Oggi, del Teatro, I982, p. 462, source très sûre, donne cette date; Campardon et Léris donnent la même date.

7. P. 255. Pascariel était joué vers 1685 par Joseph Torteriti, qui joua ensuite Scaramouche en I694 (d'Origny et Campardon).

8. Biancolelli avait épousé la fille de Pascariel à Grenoble en I703, et ses relations avec la ville de Grenoble étaient plus anciennes qu'il ne paraît.

9. www.cesar.org.uk. Ce répertoire établi par Barry Russel année par année à partir de Parfaict, Campardon, Léris, Brenner, Conlon, etc., est très complet. L'ouvrage le plus informé sur l'histoire de la troupe de Biancolelli est celui de Léon Vallas, Un siècle de musique et de théâtre à Lyon. I688-1789, Lyon, Masson, 1932.

Io. Les relations entre Biancolelli et Barbier ont été étudiées avec précision par J. RittaudHutinet et M. Pruner dans leur édition de La Promenade des Terreaux de Lyon (Centre d'études et de recherches théâtrales de l'université Lyon 2, préface de G. Couton, 1977), étude très informée dont nous utiliserons plus complètement les données dans notre édition. 
célèbre dans le rôle d'Arlequin, hérité de son père, et d'après Léris, le régent l'aurait appelé à Paris comme remplaçant éventuel de Thomassin. On en déduira que dans Les Plaisirs de La Tronche, il jouait le personnage d'Arlequin. Le personnage de Colombine a sans doute été tenu par Jeanne Jacquette, femme de Dominique et titulaire du rôle. Le reste de la troupe de I7II était français, de naissance, de langue ou de formation, même si les rôles étaient italiens: Léandre, Arlequin et Pierrot, Isabelle et Colombine, Fédéric, Scaramouche, Octave, le docteur sont des personnages de la commedia dell'arte. Sont certainement français aussi les bergers et bergères, les musiciens et la chanteuse, Mlle de Saint-Jacques; sans doute venaient-ils de cette Académie royale de musique de Lyon qui donnait, trois ans plus tôt à Grenoble, La Fée bienfaisante II. Les Italiens de I7II, établis en France depuis au moins quarante ans, et souvent nés en France, parlaient français, contrairement à la troupe de I7I6, mais ils restaient fidèles à la comédie de lazzi; cela explique le style particulier des Plaisirs de La Tronche, cette pièce française jouée à l'italienne.

Dans sa préface, L. B. est visiblement gêné aux entournures; il comprend mal cette troupe italienne "dont le caractère [lui] est assez inconnu»; il sait que «la beauté de leur jeu est dans le geste $\&$ la manière dont ils débitent ce qu'ils disent», mais ignore le genre de leur comédie. On doit donc supposer que toutes les scènes où les acteurs rivalisent de fantaisie dans l'improvisation gestuelle ou verbale selon la meilleure tradition italienne, ne sont pas de lui, mais d'un connaisseur de la commedia dell'arte; or cela fait déjà plus de la moitié de la pièce; et comme la construction de la comédie repose en fait sur tous les pièges tendus par les "Italiens" à un jaloux grenoblois, on s'interrogera un peu plus encore sur la part qui revient à La Baume dans l'écriture de la pièce. Peut-être cette part doit-elle être réduite à l'argument initial. Pressé par le temps, La Baume s'est en effet replié sur un thème italien traditionnel, celui de la jalousie morbide:

Comme on soupçonne ordinairement cette nation de jalousie, je fais paraître le principal Acteur jaloux d'une fille déguisée en homme pour en rendre par là le dénouement plus agréable $[\ldots]$

La jalousie italienne, telle qu'elle apparaissait dans les mémoires, les romans et les nouvelles depuis Boccace et Bandello, était sombre et tragique; dans la comédie, on se retrouve plutôt dans les traces de Dandin, d'Arnolphe, de Sganarelle et d'Alceste, car le problème est de faire rire. Voici donc Léandre, époux amoureux possessif, ombrageux, de la belle et coquette Isabelle; on

II. Il existait à Grenoble une Académie royale de musique, attestée dès 1725, mais pour des fêtes religieuses données dans la cathédrale, alors que l'Académie de Lyon donnait des opéras et semble avoir suivi les Italiens dans leur tournée. 
reconnait de place en place Alceste et Célimène, mais Isabelle est ici une charmante épouse qui aime simplement la compagnie et s'irrite un peu de la méfiance de son mari; aussi pour le punir a-t-elle imaginé de déguiser sa jeune nièce en un brillant cavalier qui affectera de lui faire une cour pressante; et Léandre est furieux. On rira donc de ses fureurs, sans doute justifiées, mais excessives et déplacées. Tel est le rire français, qui procède d'un jugement: en France, on est raisonnable, modéré, sociable, on sait aimer avec délicatesse et générosité, on se garde d'être jaloux. On se moque des mauvaises manières, italiennes, plus souvent espagnoles, bientôt anglaises. Ces gens-là font l'amour tristement; qu'est-ce que la jalousie sinon cette «amitié goulue, qui n'en veut que pour soi ${ }^{12}$ »? Molière l'avait dit. Le débat était à la mode dans les années I670. L'auteur des Plaisirs le reprend un peu tard et pour plaire à la bonne société de Grenoble, flattée d'illustrer ainsi le bon ton. Il n'hésite pas à énoncer ici et là les principes de la vie en société: «une personne de bon esprit se divertit de tout sans donner dans aucun excès" (sc. VIII), se partage entre la ville et la campagne, entre les visites et le jardinage. Et puisqu'Isabelle est un modèle de "bonne éducation", elle sait énumérer les plaisirs délicats:

[...] parties de campagne au moins une fois la semaine, des conversations libres, éveillées \& modestes, des petits jeux d'amusements, des défis en vers, en chansons, en poulets, en énigmes, apprendre des comédies, ensuite les jouer ensemble. (sc. VIII)

C'est elle, en somme, qui définit le programme innocent des «plaisirs de La Tronche». Nous en sommes encore à la conversation de salon: la ville ou la campagne? la jalousie à l'italienne ou la tendre sollicitude à la française? On conçoit que notre Léandre italien jouera plutôt dans le registre des barbons, des ours mal léchés, de Dandin ou d'Arnolphe:

$[\ldots]$ je vois ce que je vois, malheureux que je suis! où me suis-je engagé, mais enfin je l'ay voulu, il faut m'en tirer comme je pourrai \& prendre des mesures pour cela. (sc. I)

Son jeu est statique: il pratique volontiers le soliloque, s'interroge sur la conduite à tenir, sur sa femme, qu'il ne peut s'empêcher d'aimer: il serait plus tranquille si elle était laide, mais fallait-il épouser une laide pour avoir la paix? Le soliloque devient tragique dès qu'il se croit trahi pour de bon:

Qu'ay-je entendu grand Dieu? ô ciel est-il possible qu'on pousse la trahison \& l'effronterie jusquà ce point-là? (sc. XVII)

Pour nous rappeler que la scène est comique, il faudra alors le dépeindre «seul, sortant de son coin comme en furie, sa perruque de travers». Il faudra

I2. L'École des femmes, II, 2, v. 443-444 (Alain). 
aussi en faire un atrabilaire amoureux, un Alceste qui rêve d'entraîner sa femme dans le désert: «mais je les attraperay tous, il faut absolument que je la mène à la campagne, \& ainsi leur rompre à tous en visière, morbleu ", et il "frappe du pied» (sc. IV). L'emmener très loin dans sa "campagne», cela veut dire en effet qu'il faut aller plus loin que La Tronche, plus loin que "L'Ilevert» ou Vizille: il faut prendre à la Messagerie une voiture et aller, qui sait? dans le Grésivaudan. J'ouvre une parenthèse: L. B. et Dominique, dira-t-on, connaissent bien la région. On peut pourtant avoir un doute : à Lyon, Dominique joue La Promenade des Terreaux ou La Diligence de Lyon, à Rennes La Promenade de Rennes, à Bordeaux La Promenade des fossés. Ce régionalisme ne lui coûte guère ${ }^{13}$. Cependant Léandre est de plus en plus livré à ses noires vapeurs et à ses idées fixes: Isabelle se serait-elle enfermée avec Federic dans le labyrinthe (sc. X) ? Pierrot les espionnera pour dix pistoles (sc. XII) et en donnera à Léandre pour son argent; le jaloux va se cacher dans un coin et écouter ce qu'ils disent; prévenus, Isabelle et Federic lui jouent une scène d'amour à vrai dire un peu osée:

Federic: Ha, permettez que je la baise, cette bouche charmante.

Isabelle: Quoi, d'abord vous vous payez par vos mains?

Federic: Quelles douceurs, grand Dieu, jamais lèvres ne furent si appétissantes, $\&$ quand je devrais être grondé, je veux encore vous en voler un second.

Les amants supposés ne sont plus maîtres de leurs "transports», se jurent un amour éternel et se donnent rendez-vous pour le soir; Léandre est fou de jalousie; on le serait à moins: la tante et la cousine y vont un peu fort. Comme on l'aura compris, dans Les Plaisirs de La Tronche, le rire français est un rire jaune; il naît de l'excès, de l'exclusion du malade et de plaisanteries traditionnelles sur le cocu malgré lui.

Quand Dominique a vu ce scénario et cette version noire du Léandre amoureux, il a dû se demander ce qu'il en ferait. Or il en fait, comme on pouvait l'attendre, une pièce italienne, dans laquelle il se donne le beau rôle, celui de l'irrésistible Arlequin qui, en serviteur glouton, gaffeur et gesticulateur, devient le double burlesque de Léandre, ce qui change tout. Il est glouton comme Léandre est amoureux, sans mesure, sans limite, livré comme lui à une idée fixe: celle de la nourriture et du vin. Le parallèle est exploité dès la scène $\mathrm{V}$ :

Léandre: Hé bien, où est ma Femme?

Arlequin: Monsieur, elle va souper à La Tronche, c’est Federic qui régale [...] le souper est déjà parti.

I3. Je note au passage une petite allusion à la rue du Palais, redoutée d'Arlequin (à cause du Palais de justice), alors qu'il aime la grande rue (p. 15). Cela ne nous mène pas loin. 
Et tandis que Léandre ne pense qu'à la voiture qui emmènera Isabelle loin de La Tronche, Arlequin détaille le menu et se voit déjà à table, à La Tronche: c'est drôle, et le contraste a une réelle valeur scénique. L'opposition se poursuit et s'accentue dès que le serviteur est au fait du complot et se met à trahir délibérément son maitre: pour l'amour du repas, du vin et des pistoles, il égare Léandre, dont il cultive les fantasmes, dans son propre style, ainsi pour le rendez-vous du soir:

Isabelle a répondu: je le veux bien, mon cher petit Poulet, j'iray partout où vous voudrez. Ah ma Reine, a-t-il répondu, que vous êtes aimable, allons-nous en dans la chambre de l'alcôve, \& là nous serons tranquilles: Et je suis venu, Monsieur, vous en avertir, \& vous demander mes dix pistoles. (sc. XV)

Pour enrichir encore le parallèle par opposition entre le maître et le serviteur, il suffira de rendre à son tour Arlequin amoureux et jaloux; une autre comédie s'annonce alors en parallèle burlesque: Arlequin va "paroliser» avec son maître, c'est-à-dire renchérir sur lui, comme au paroli; une scène de dépit s'esquisse, elle ne durera pas plus que la scène XIII et se résume en une série de lazzi, mais on comprend que toute la comédie repose en fait sur une surenchère et un contrepoint continuel entre les deux formes de comique. Léandre était immobile, renfrogné, enfermé en lui-même, Arlequin sera gesticulateur, exubérant et extraverti. Si l'un parle seul, l'autre s'adresse au public entier; si l'un menace, l'autre se confond en courbettes, prosternations et dérobades, gambades et fous rires (sc. XII). Tel est le rire italien : il est à base de parodies et d'imitations, puis de pure pantomime et de lazzi. Arlequin parodie son maître, mais peut aussi bien parodier Pierrot ou Scaramouche, Colombine ou Mezzetin: c'est le jeu à deux. La scène IV, de la clé et du bâton entre Arlequin et Scaramouche, en est un bon exemple, mais plus encore la scène de "mines" entre Arlequin et Colombine (sc. XIII). Il peut aussi jouer seul, dans une enfilade de lazzi. Les Plaisirs de La Tronche en comptent près d'une dizaine, qui appartiennent pour la plupart au répertoire d'Arlequin; ils sont le plus souvent signalés en didascalie: Arlequin après plusieurs postures pour chercher à qui parle son Maître, et ne voyant personne, dit [...] (sc. I); Il rit \& fait plusieurs lazzi là-dessus, \&o tous ses lazzi étant finis, il voit tout à coup Colombine devant lui [...] (sc. XII). Le plus souvent, le texte fait simplement allusion au jeu des acteurs: Ils font icy des lazzi qui marquent la joye quills ont [...] Ils sortent en faisant des lazzi de cérémonie (sc. XIII). Très souvent, on devine que les acteurs se livrent à des «lazzi de sortie», simulant l'excès de politesses, ou se bousculant et faisant "semblant de battre quelqu'un : «ils sortent ainsi du théâtre comiquement» (sc. XX). Ces lazzi, n'étant pas écrits, laissent en fait carte blanche à l'acteur: Arlequin peut longuement simuler un repas (sc. v) ou «contrefaire l'ivre» (sc. XXII) aussi longtemps que le public rira, ou se complaire en 
allusions grivoises: "Il fait quelques lazzi en disant, jaloux d'une fille?» (sc. XXII). On pourrait penser que ces lazzi à répétition font de la pièce un simple canevas. On remarque toutefois qu'ils s'enchainent sans jamais s'écarter beaucoup du thème de l'intrigue: la jalousie, ou la goinfrerie, également possessives. C'est pourquoi l'on peut parler de contrepoint, et c'est pourquoi aussi l'on est tenté de l'attribuer à un seul auteur, qui a toutes les chances d'être Dominique: la pièce garde sa logique et son développement. Elle parvient à conserver un équilibre entre ses trois composantes scéniques: le dialogue classique, l'improvisation gestuelle, la musique enfin. Ce sont des constantes du théâtre de Biancolelli, qui compte une bonne centaine de comédies, dont près de la moitié ont été publiées.

Sans doute s'agit-il d'une "partie de campagne» faite pour un théâtre de société: Biancolelli ne l'a pas recueillie dans son Nouveau Théatre italien de I7I3, peut-être parce qu'il l'avait composée sur commande pour le compte de La Baume, peut-être parce qu'à la veille de se produire à Paris, il ne voulait pas se prévaloir de cette petite pièce en prose aux couplets assez lestes. Sommé d'écrire en quelques jours un petit acte avec Italiens et musique, il s'est assez bien tiré d'affaire. Il connaît son Molière par cœur, il compose un petit Misanthrope en un acte, et qui finit bien. Cette pièce française, il l'habille à l'italienne. Biancolelli, qui a été nourri de théâtre dès son enfance, a eu vite fait de voir le parti qu'il pouvait tirer de cet argument un peu maigre, et soit qu'il ait travaillé seul, soit qu'il ait rassemblé après coup dans ses didascalies les improvisations de ses coéquipiers ${ }^{\mathrm{I}}$, une pièce est là sur le papier. Il disposait d'un Léandre, d'un Arlequin et d'une Colombine de valeur, d'un Scaramouche peut-être, ou d'un Mezzetin's, et d'un Docteur; il leur a fait la part belle. On pourrait imaginer que d'autres acteurs aient été des amis de L. B. ou de la Présidente, des amateurs grenoblois: Isabelle, Federic, Octave, Leonore - qui n'est pas même inscrite sur la liste des personnages - ont des rôles de simples mondains et n'ont droit ni aux lazzi ni aux mines; mais la troupe de Dominique, qui se déplaçait avec ses douze acteurs, ses musiciens, sa chanteuse et son décorateur, n'avait pas besoin d'aide. Seul Pierrot, qui jure par «morbleu», "par le sanguié» et "pardie» a l'air d'être du pays; curieusement, il intervient avec une Thievena pour une scène en patois dauphinois, haute en couleur ${ }^{16}$ (sc. XIX). On a donc utilisé tous les acteurs qu'on avait sous la

I4. Le texte imprimé a été réalisé à la diable; le rédacteur, qui est peut-être simplement le souffleur, paraît mal connaître l'italien: le Ohimé, devient sous sa plume «Ho-ymet» ou «hai met» (sc. XV, XX).

I5. Dans la scène XX, le nom de Scaramouche est tout à coup remplacé par celui de Mezzetin, comme si le même acteur jouait ordinairement les deux rôles.

I6. Cette scène dauphinoise, dans un dialecte francoprovençal dont G. Tuaillon nous a 
main avec un certain bonheur. On disposait aussi d'une troupe de danseurs, de musiciens, et d'une chanteuse, $M^{\text {lle }}$ Saint-Jacques. Dès la fin de la scène VII, «le Théâtre change \& laisse voir le costeau de La Tronche», l'orchestre, une "bande de violons», se rassemble et accompagne $\mathrm{M}^{\text {lle }}$ Saint-Jacques; on ne sait ce qu'elle chante; on soupçonne qu'elle est venue de Lyon avec son répertoire. À la fin de la pièce, bergers et bergères font une entrée sur un air de marche, on joue deux menuets en ballet, puis un rigodon, et chacun y va de son couplet sur les plaisirs de La Tronche: Saint-Jacques et Arlequin-Dominique en premier; puis sur l'air du menuet, $M^{\text {lle }}$ Saint-Jacques et Pierrot, et enfin la "compagnie» en chœur. La pièce commençait en comédie classique, elle s'est continuée en commedia dell'arte, elle s'achève en opéra comique comme à la Foire: en une demi-heure, elle nous fait parcourir les genres les plus appréciés au début du siècle, ceux que les comédiens italiens ont pratiqués tour à tour ou tout ensemble pendant vingt ans. Elle ne quitte pas pour autant son objet, qui est de nous décrire les Plaisirs: plaisirs français de la vie en société, des divertissements mondains et d'un marivaudage un peu cruel; plaisirs italiens de la bonne chère, de la danse et des gambades. La Tronche, nouvelle Cythère, à la fois pastorale et libertine, rassemble tous les plaisirs de la vie et tous les rires:

Si vous voulez, bergères,

Narguer les plus jaloux,

Quittez vos airs sévères,

Risquez un rendez-vous,

La Tronche est un asile

Où l'on peut sûrement

Tromper le plus habile,

Et rendre heureux l'amant.

Rire français, rire italien? Biancolelli, comme Watteau, comme Couperin à la même époque, célèbre les goûts réunis ${ }^{17}$.

garanti l'authenticité, fait exception dans la pièce, et Thievena (Étiennette) ne fait pas d'autre apparition. Pierrot, lui, a les honneurs du finale. Il se peut qu’il ait joué les rôles de paysan et qu'il ait assimilé rapidement l'accent dauphinois.

17. Couperin travaille à son premier livre de clavecin (avec une pièce sur «les plaisirs de Saint-Germain-en-Laye»); Watteau peindra en I718 le grand Gilles, qui est sans doute un Pierrot, joué peu de temps auparavant par Biancolelli à la foire Saint-Laurent (voir F. Moureau, "Watteau et son temps», dans le catalogue de l'exposition Watteau de 1984, Paris, éd. de la Réunion des Musées nationaux, p. 490). 